\title{
Primary neurocritical care involving therapeutic hypothermia for acute ischemic stroke patients with malignant infarct cores
}

\section{ORIGINAL ARTICLE \\ Journal of Neurocritical Care \\ Received: April 1, 2019 \\ Revised: April 30, 2019 \\ Accepted: May 12, 2019 \\ Corresponding Author: \\ Ji Man Hong, MD, PhD \\ Department of Neurology, \\ Ajou University School of Medicine, 164 World cup-ro, Yeongtong-gu, Suwon 16499, Republic of Korea \\ Tel: +82-31-219-5175 \\ Fax: +82-31-219-5178 \\ E-mail: dacda@hanmail.net}

Seong-Joon Lee, MD, PhD; Kyu Sun Lee, MD; Jin Soo Lee, MD, PhD; Mun Hee Choi, MD; Sung Eun Lee, MD; Ji Man Hong, MD, PhD

Department of Neurology, Ajou University School of Medicine, Suwon, Republic of Korea

\begin{abstract}
Background: Acute ischemic stroke patients with malignant infarct cores were primarily treated with neurocritical care based on reperfusion and hypothermia. We evaluated the predictors for malignant progression and functional outcomes.

Methods: From January 2010 to March 2015 ischemic stroke patients with large vessel occlusion of the anterior circulation with infarct volume $>82 \mathrm{~mL}$ on baseline diffusion weighted image (DWI) within 6 hours from onset, with National Institutes of Health Stroke Scale $\geq 15$ were included. All patients were managed with intent for reperfusion and neurocritical care. Malignant progression was defined as clinical signs of progressive herniation. Predictive factors for malignant progression and outcomes of decompressive hemicraniectomy (DHC) were evaluated.

Results: In total, 49 patients were included in the study. Among them, $33(67.3 \%)$ could be managed with neurocritical care and malignant progression was observed in the remainder. Decompressive surgery was performed in nine patients (18.4\%). Factors predictive of malignant progression were initial DWI volumes (odds ratio $[\mathrm{OR}], 1.01 ; 95 \%$ confidence interval $[\mathrm{Cl}], 1.00$ to 1.02; $P=0.046)$ and parenchymal hematoma $(O \mathrm{R}, 6.77 ; 95 \% \mathrm{Cl}, 1.50$ to $30.53 ; P=0.013)$ on computed tomography taken at Day 1 . Infarct volume of $>210 \mathrm{~mL}$ predicted malignant progression with $56.3 \%$ sensitivity and $90.9 \%$ specificity. Among the malignant progressors, 77.7\% resulted in grave outcomes even with DHC, while all patients who declined surgery died.

Conclusion: Acute ischemic stroke patients with malignant cores between 82 to $209 \mathrm{~mL}$ can be primarily treated with neurocritical care based on reperfusion and hypothermia with feasible results. In patients undergoing surgical decompression due to malignant progression, the functional outcomes were not satisfactory.
\end{abstract}

Keywords: Infarction, middle cerebral artery; Brain edema; Thrombectomy; Hypothermia, induced; Critical care; Decompressive craniectomy

\section{INTRODUCTION}

The combined analyses of decompressive hemicraniectomy (DHC) trials have shown that in patients with malignant middle cerebral artery (MCA) infarction, DHC undertaken within 48 hours of stroke onset reduces mortality and increases the number of patients with a favorable functional outcome [1]. However, since the success of DHC trials, there have been radical changes in the

(C) 2019 The Korean Neurocritical Care Society

This is an Open Access article distributed under the terms of the Creative Commons Attribution Non-Commercial License (http://creativecommons.org/licenses/by-nc/4.0/) which permits unrestricted noncommercial use, distribution, and reproduction in any medium, provided the original work is properly cited. 
field of acute stroke. In terms of reperfusion, intravenous (IV) thrombolysis has been approved for the time window of up to 4.5 hours [2], and endovascular treatment (EVT) has become the standard of care [3]. Neurocritical care for patients with massive ischemic stroke has also improved, especially in terms of neuroprotection and the antiswelling effect of therapeutic hypothermia [4]. While the therapeutic benefits of hypothermia on functional outcomes need to be further validated in prospective trials [5], there is evidence that it can reduce cerebral edema, reduce hemorrhagic transformation and improve outcomes, especially in those with complete reperfusion $[4,6]$. These developments have possibly increased the number of patients eligible for reperfusion therapies, while potentially reducing the number of patients that show malignant progression [7].

Furthermore, in acute stroke populations eligible for reperfusion, DHC trials could not be applied, as randomization usually occurred at least 12 hours after symptoms onset [8], and usually did not include patients in the acute period who underwent IV thrombolysis [9]. Accordingly, there are currently few indicators for the optimal management of acute stroke patients with large vessel occlusion presenting with malignant infarct cores.

For such patients, our institution pursues rapid reperfusion, combined with primary neurocritical care based on therapeutic hypothermia. The impact of malignant progression and its predictive factors have not been studied in this population. Furthermore, the outcomes of emergent $\mathrm{DHC}$ in patients primarily managed with neurocritical care have not been reported. Therefore, the aim of our study was to address these points.

\section{METHODS}

\section{Patient enrollment}

This was a retrospective single center study from January 2010 to March 2015. In this period, 315 patients underwent our institutional stroke endovascular reperfusion critical pathway. Of these patients, those with acute ischemic stroke involving the MCA territory with infarct volume $>82 \mathrm{~mL}$ on baseline diffusion weighted image (DWI) who presented within 6 hours from onset [10], with National Institutes of Health Stroke Scale (NIHSS) $\geq 15$ (according to previous literature that malignant cerebral edema is seldom encountered in patients with NIHSS under 15) [11] were included. Infarct size was measured in baseline DWI using nordicICE semiautomated software (NordicNeuroLab, Bergen, Norway). Patients with significant contralateral infarction or space occupying intrainfarct hemorrhage were excluded.

The data collection protocol was approved by the Institutional Review Board of each participating hospital and implemented in ac- cordance with the ethical standards of the 1964 Declaration of Helsinki and its later amendments. The need for written informed consent was waived given the retrospective nature of the study.

\section{Reperfusion treatment}

IV thrombolysis and endovascular reperfusion treatment were performed on patients based on the decision of the attending stroke neurologist. All patients underwent cerebral angiography for the initial intent of mechanical thrombectomy. In patients with very large DWI infarct volumes, aggressive reperfusion was avoided after performing digital subtraction angiography. However, as most of the patients were managed before the success of major EVT trials, and there is still disagreement about the upper infarct volume limit for EVT, reperfusion was sometimes performed for very large infarcts.

\section{Primary neurocritical care}

All patients were managed with intent for primary neurocritical care. This was performed based on a stepwise protocol, and the treatment degree was individualized on a patient by patient basis [12]. In brief, the treatment starts with general management including head elevation, easy venous drainage, and avoidance of fever, hypoxia, hypercapnia, or hyponatremia. Early rapid sequence intubation was performed on patients with altered mental status, or those in which therapeutic hypothermia was performed. In patients who underwent hypothermia, cooling was performed with adequate sedation and neuromuscular blockade. Patients underwent 48 hours of cooling (target temperature $34.5^{\circ} \mathrm{C}$ ) and 48 hours of rewarming. Osmotherapy was performed with mannitol or hypertonic saline as required.

\section{Malignant progression and DHC}

Late DHC was usually performed based on clinical signs. Throughout neurocritical care, clinical signs of progressive herniation such as significant decrease in mentality, unilateral pupillary abnormality, impairment of eye movements, respiratory pattern abnormalities, and flexor or extensor motor posturing [13] were screened. Patients that showed these clinical signs, with computed tomography (CT) evidence of impending or imminent herniation, were termed as malignant progressors. DHC was performed on these patients as soon as possible after clinical decision and consent. There were no age limitations for decompressive surgery if family agreement was obtained. Previous IV thrombolysis was not a contraindication for decompression in this study. It was not performed on patients with bilateral fixed pupils [1].

\section{Statistical analysis}

First, the patients were dichotomized to malignant progressors and 
nonprogressors. Factors predictive of malignant progression were evaluated through univariate and multivariate analysis. Second, the impact of initial DWI volume on prediction of malignant change was evaluated through receiver operating characteristic analysis. The functional outcomes of DHC performed on malignant progression were evaluated. A grave outcome was defined as a modified Rankin Scale (mRS) of 5-6. Third, factors predictive of grave outcomes in the patients that were managed with primary neurocritical care were evaluated through univariate and multivariate analysis. The data are presented as the mean \pm standard deviation or as the median (interquartile range). A $P<0.05$ was considered statistically significant. For multivariate analysis, variables with $P$ values $<0.100$ were included for logistic regression analysis by the forward conditional method. Statistical analyses were performed using IBM SPSS Statistics software version 25 (IBM Corp., Armonk, NY, USA).

\section{RESULTS}

\section{Overall outcomes}

A total of 49 patients were included for analysis in this study. IV thrombolysis was performed in 37 patients (75.5\%) and EVT was performed in 39 patients (79.6\%). Any form of reperfusion was performed in 45 patients (91.8\%), while both IV thrombolysis and EVT was performed in 31 patients (63.3\%). Therapeutic hypothermia was performed in 27 patients (55.1\%). Among the 49 patients, $33(67.3 \%)$ could be managed with primary neurocritical care, without malignant progression. In the nonprogressors, 3-month functional outcomes of mRS 0-3 were achieved in $27.3 \%$, while grave outcomes were recorded in $42.4 \%$. However, among the malignant progressors, $87.5 \%$ resulted in grave outcomes (Table 1).

Table 1. Factors predictive of malignant progression

\begin{tabular}{|c|c|c|c|c|c|}
\hline Variable & Nonprogressor $(n=33)$ & Malignant progressor $(n=16)$ & Univariate $P$ value & OR $(95 \% \mathrm{Cl})$ & Multivariate $P$ value \\
\hline \multicolumn{6}{|l|}{ Clinical profile } \\
\hline Age (yr) & $69.5 \pm 13.3$ & $65.1 \pm 17.9$ & 0.335 & & \\
\hline Male sex & $16(48.5)$ & $8(50.0)$ & 0.921 & & \\
\hline HTN & $20(60.6)$ & $13(81.3)$ & 0.148 & & \\
\hline DM & $7(21.2)$ & 7 (43.8) & 0.101 & & \\
\hline Dyslipidemia & $16(48.5)$ & $11(68.8)$ & 0.362 & & \\
\hline Smoking & $5(15.2)$ & $5(31.3)$ & 0.190 & & \\
\hline Atrial fibrillation & $20(60.6)$ & $6(37.5)$ & 0.201 & & \\
\hline Initial NIHSS & $18.0(15.0-19.5)$ & $17.5(16.0-20.5)$ & 0.200 & & \\
\hline \multicolumn{6}{|l|}{ Infarct burden } \\
\hline DWI volume & $149 \pm 44$ & $236 \pm 114$ & 0.009 & $1.01(1.00-1.02)$ & 0.046 \\
\hline \multicolumn{6}{|l|}{ Therapeutic management } \\
\hline Thrombolysis & $26(78.8)$ & $11(68.8)$ & 0.444 & & \\
\hline EVT & $23(69.7)$ & $16(100.0)$ & 0.014 & - & - \\
\hline Mannitol & $20(60.6)$ & 15 (93.8) & 0.016 & - & - \\
\hline Hypertonic saline & $2(6.1)$ & $3(18.8)$ & 0.169 & & \\
\hline Mechanical ventilation & $21(63.6)$ & $14(87.5)$ & 0.083 & - & - \\
\hline Hypothermia & $18(54.5)$ & $9(56.3)$ & 0.910 & & \\
\hline Hemorrhagic change on D1 CT & & & 0.003 & & \\
\hline None & $11(33.3)$ & $1(6.3)$ & & & \\
\hline $\mathrm{HI} 1$ & $6(18.2)$ & $2(12.5)$ & & & \\
\hline $\mathrm{HI} 2$ & $9(27.3)$ & $1(6.3)$ & & & \\
\hline $\mathrm{PH} 1$ & $4(12.1)$ & $3(18.8)$ & & & \\
\hline $\mathrm{PH} 2$ & $3(9.1)$ & $9(56.3)$ & & & \\
\hline $\mathrm{PH} 1-2$ & $7(21.2)$ & $12(75.0)$ & $<0.001$ & $6.77(1.50-30.53)$ & 0.013 \\
\hline \multicolumn{6}{|l|}{ Functional outcome } \\
\hline mRS 0-2 & $6(18.2)$ & 0 & 0.069 & & \\
\hline $\mathrm{mRS} 0-3$ & $9(27.3)$ & $1(6.3)$ & 0.087 & & \\
\hline mRS 5-6 & $14(42.4)$ & $14(87.5)$ & 0.003 & & \\
\hline
\end{tabular}

Values are presented as mean \pm SD, number (\%), or median (interquartile range). Variables with $P$ values $<0.100$ in univariate analysis were included for multivariate analysis. Variables included in multivariate analysis but not included in the final model are marked with a hyphen.

OR, odds ratio; HTN, hypertension; DM, diabetes mellitus; NIHSS, National Institutes of Health Stroke Scale; DWI, diffusion-weighted imaging; EVT, endovascular therapy; $\mathrm{CT}$, computed tomography; $\mathrm{HI}$, hemorrhagic infarct; $\mathrm{PH}$, parenchymal hematoma; mRS, modified Rankin Scale. 


\section{Prediction of malignant changes}

in the analysis to detect potential factors predictive of malignant progression, initial DWI lesion volume (odds ratio [OR], 1.01; $95 \%$ confidence interval $[\mathrm{CI}], 1.00$ to $1.02 ; P=0.046)$ and parenchymal hematoma, postprocedure or at Day 1, by CT (OR, 6.77; $95 \% \mathrm{CI}, 1.50$ to $30.53 ; P=0.013$ ) showed significant association, highlighting the impact of initial infarct volume on malignant progression (Table 1). EVT, or IV osmotherapy with mannitol, was associated with malignant progression in the univariate analysis, but this association was not apparent in the multivariate analysis.

When the predictive ability of initial DWI for malignant progression was assessed, it could predict malignant course with an area under the curve of 0.735 (range, 0.569 to 0.901 ) (Fig. 1). Infarct volume of $>210 \mathrm{~mL}$ predicted malignant progression with $56.3 \%$ sensitivity and $90.9 \%$ specificity. When the included patients were divided into subgroups according to initial infarct volume, the percentage of malignant progressors increased sharply when the infarct volume increased to $>210 \mathrm{~mL}$, with $42.9 \%$ progressors in the 201 to $299 \mathrm{~mL}$ group, while all patients with $>300 \mathrm{~mL}$ of infarct core showed malignant progression (Fig. 1).

\section{Impact of decompressive surgery}

among the 16 malignant progressors, nine underwent DHC and seven refused surgery. In the nonprogressors, grave outcomes were encountered in $42.4 \%$ of patients. However, of the malignant progressors, $87.6 \%$ resulted in grave outcomes $(P=0.031)$ (Fig. 2). Even with DHC, $77.7 \%$ resulted in grave outcomes, while all patients that declined surgery died.

\section{Outcomes in the nonprogression group}

When the factors predictive of grave outcomes in the nonprogressors were delineated, age (OR, 1.08; 95\% CI, 1.01 to $1.16 ; P=0.024)$ and initial DWI volume (OR, 1.03; 95\% CI, 1.00 to $1.05 ; P=0.049)$ were shown to be significant predictive factors (Table 2). Performing EVT was associated with $\mathrm{mRS} 0-4$ in the univariate analysis, but this association was insignificant in the multivariate analysis. There was a tendency for therapeutic hypothermia, resulting in more frequent $\mathrm{mRS}$ $0-4(P=0.062)$.

\section{DISCUSSION}

The results of this study show that primary neurocritical care with reperfusion and therapeutic hypothermia is feasible for acute ischemic stroke with malignant infarct cores. Factors predictive of malignant transformation are presenting infarct volume and occurrence of parenchymal hematoma. An infarct volume $>210 \mathrm{~mL}$ was associated with increased ratios for malignant course. For those patients in which malignant progression occurred, decom-
A

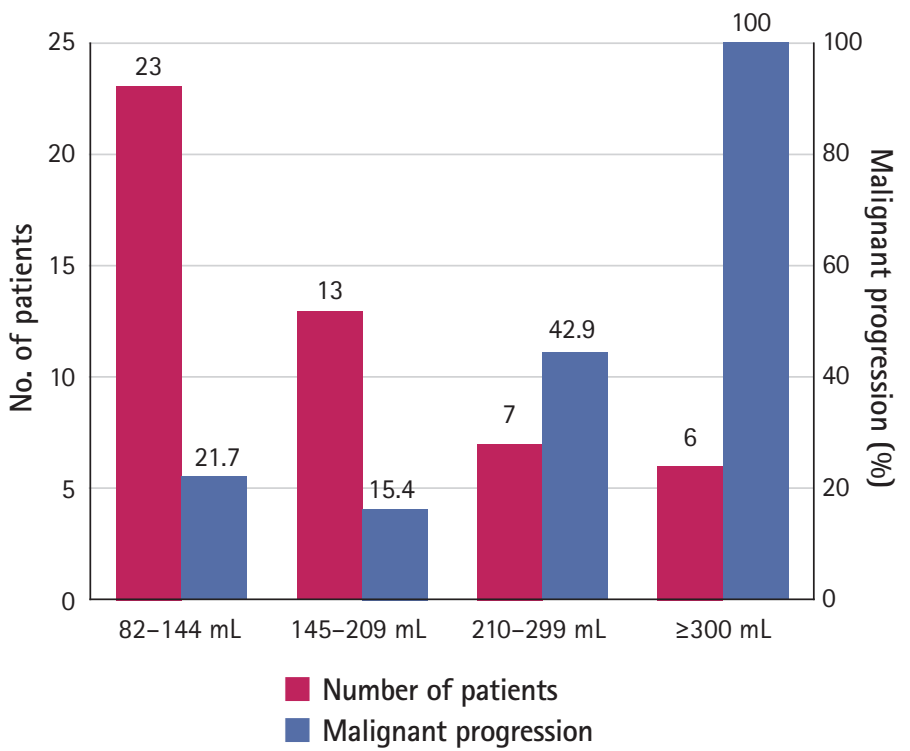

B

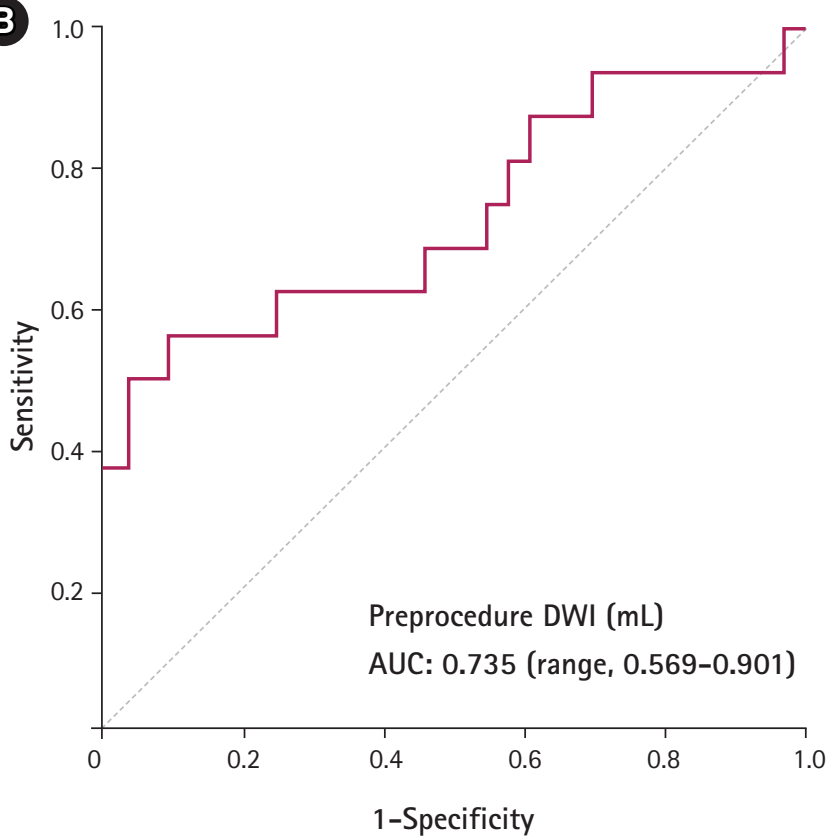

Fig. 1. Distribution of initial diffusion weighted imaging (DWI) volumes and receiver operating characteristic (ROC) curve in prediction of malignant change. (A) The percentage of patients with malignant progression sharply increases with initial DWI volumes of $>210 \mathrm{~mL}$. (B) For prediction of malignant progression with initial infarct volume, the area under the ROC curve is 0.735 , and an infarct volume threshold of $>210 \mathrm{~mL}$ predicted malignant progression with $56.3 \%$ sensitivity and $90.9 \%$ specificity. AUC, area under the curve. 


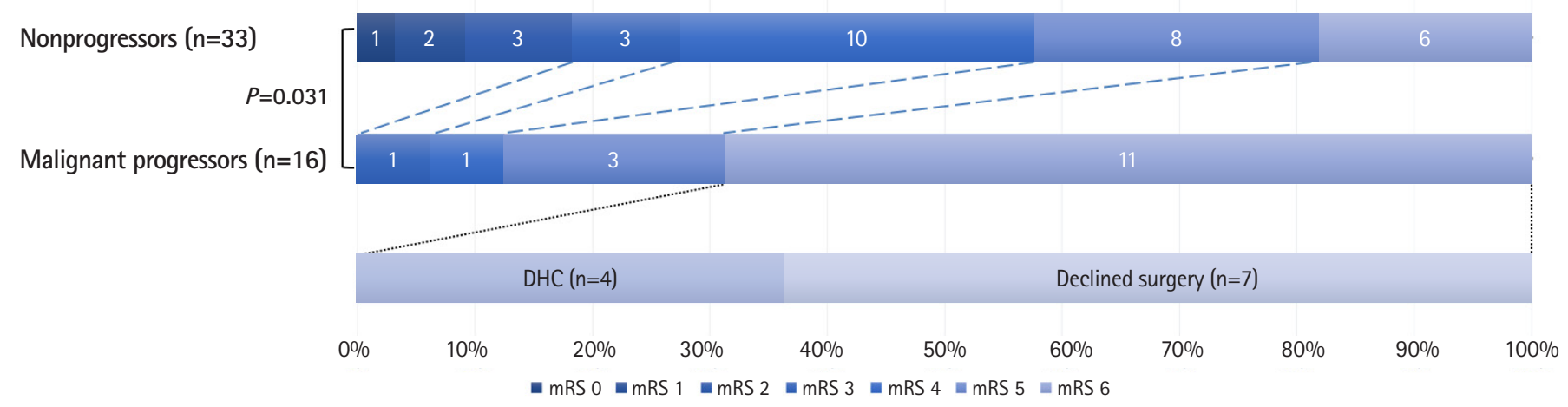

Fig. 2. Functional outcomes of nonprogressors and malignant progressors. DHC, decompressive hemicraniectomy; mRS, modified Rankin Scale.

Table 2. Factors predictive of outcomes in the nonprogressors

\begin{tabular}{|c|c|c|c|c|c|}
\hline Variable & mRS 0-4 $(n=19)$ & mRS 5-6 $(n=14)$ & Univariate $P$ value & OR $(95 \% \mathrm{Cl})$ & Multivariate $P$ value \\
\hline \multicolumn{6}{|l|}{ Clinical profile } \\
\hline Age (yr) & $65 \pm 14$ & $76 \pm 8$ & 0.014 & $1.08(1.01-1.16)$ & 0.024 \\
\hline Male sex & $12(63.2)$ & $4(28.6)$ & 0.049 & - & - \\
\hline HTN & $11(57.9)$ & $9(64.3)$ & 0.710 & & \\
\hline DM & $2(10.5)$ & $5(35.7)$ & 0.080 & - & - \\
\hline Dyslipidemia & $10(52.6)$ & $6(42.9)$ & 0.530 & & \\
\hline Smoking & $4(21.1)$ & $1(7.1)$ & 0.271 & & \\
\hline Atrial fibrillation & $9(47.4)$ & $11(78.6)$ & 0.070 & - & - \\
\hline \multicolumn{6}{|l|}{ Infarct burden } \\
\hline DWI volume & $133 \pm 31$ & $170 \pm 51$ & 0.026 & $1.03(1.00-1.05)$ & 0.049 \\
\hline \multicolumn{6}{|l|}{ Therapeutic management } \\
\hline Thrombolysis & $15(78.9)$ & $11(78.6)$ & 0.979 & & \\
\hline EVT & $16(84.2)$ & $7(50.0)$ & 0.035 & - & - \\
\hline Mannitol & $10(52.6)$ & $10(71.4)$ & 0.275 & & \\
\hline Hypertonic saline & $1(5.3)$ & $1(7.1)$ & 0.823 & & \\
\hline Mechanical ventilation & $13(68.4)$ & $8(57.1)$ & 0.506 & & \\
\hline Hypothermia & $13(68.4)$ & $5(35.7)$ & 0.062 & - & - \\
\hline Hemorrhagic change on second CT & & & 0.337 & & \\
\hline None & $6(31.6)$ & $5(35.7)$ & & & \\
\hline HI1 & $2(10.5)$ & $4(28.6)$ & & & \\
\hline $\mathrm{HI} 2$ & $5(26.3)$ & $4(28.6)$ & & & \\
\hline PH1 & $4(21.1)$ & 0 & & & \\
\hline $\mathrm{PH} 2$ & $2(10.5)$ & $1(7.1)$ & & & \\
\hline $\mathrm{PH} 1-2$ & $6(31.6)$ & $1(7.1)$ & 0.090 & - & - \\
\hline
\end{tabular}

Values are presented as mean \pm SD or number $(\%)$. Variables with $P$ values $<0.100$ in univariate analysis were included for multivariate analysis. Variables included in multivariate analysis but not included in the final model are marked with a hyphen.

mRS, modified Rankin Scale; OR, odds ratio; HTN, hypertension; DM, diabetes mellitus; DWI, diffusion-weighted imaging; EVT, endovascular therapy; CT, computed tomography; $\mathrm{HI}$, hemorrhagic infarct; $\mathrm{PH}$, parenchymal hematoma.

pressive surgery did not seem to offer much benefit for improved functional outcomes, most likely due to the large infarct burden.

The primary finding of this study shows that in this population, malignant progression was a major determinant of functional outcomes. As previously reported, initial infarct volume [14] and parenchymal hematoma type hemorrhages [15] were strong predic- tors of malignant change also in this population. In contrast, endovascular reperfusion or thrombolysis was not significantly associated, showing that potential reperfusion injuries are mostly influenced by the burden of initial infarct volume.

In the malignant progressors, however, DHC did not result in the robust functional improvements seen in the DHC trials1 that 
showed $78 \%$ survival and $43 \%$ with $\mathrm{mRS} \leq 3$. This finding may be explained in two ways. First, due to the large infarct volumes (despite earlier presentations), the patients included in this study may have shown more fulminant course than those included in the decompressive craniectomy trials. This is evidenced by the concept of late window paradox [16], in which a larger core at presentation can suggest rapid growth with poor collaterals [17] or complex type occlusions [18]. Second, the patients that would have obtained functional benefit with decompressive craniectomy could have been tolerably managed with primary neurocritical care. As a result, only the patients with fulminant infarcts may have been selected for DHC and such bias could have caused the dismal outcomes in the decompressive surgery group. If this is the case, the effectiveness of reperfusion and neurocritical care on patient outcomes may diminish the potential benefits of decompressive surgery in actual clinical practice.

The findings of our study also potentially extend the volume threshold for reperfusion therapy. As compared to patients with initial infarct volume in the 82 to $144 \mathrm{~mL}$ range, patients with infarct volumes in the 145 to $209 \mathrm{~mL}$ range did not show an increased chance of malignant progression. This is in contrast to the selection criteria of infarct volumes $>145 \mathrm{~mL}$ that was used for the decompressive surgery trials [8]. Furthermore, when considering that the patients analyzed in this study presented earlier with evolving infarcts, the same $145 \mathrm{~mL}$ cut-off would include patients with potentially more malignant profiles. Thus, we can postulate that a large number of patients considered for decompressive surgery previously would have been manageable with a combination of reperfusion treatment and neurocritical care. The upper infarct volume limit of EVT has not been clearly defined and numerous studies have tried to extend the limit, which was previously taken to be an Alberta Stroke Program Early CT Score (ASPECTS) score of 6 [19]. In such studies, EVT was shown to reduce infarct growth, reduce the need for hemicraniectomy and improve outcomes in patients with ASPECTS of 5 to 7 [20]. Another study suggested a final infarct volume threshold of $133 \mathrm{~mL}$ as a cut-off for unfavorable outcomes after EVT [21]. Our study data further suggest that through the use of reperfusion therapy combined with primary neurocritical care, initial infarct volumes between 145 to $209 \mathrm{~mL}$ can be potentially manageable. Such findings warrant future prospective trials.

Our study has some limitations. First, it is limited by the retrospective nature of the study and number of patients. However, malignant cerebral edema is a rare disease with an incidence of 10 to 20 per 100,000 per year [22], and acute ischemic stroke with malignant core occurs even less often. Thus we believe that our study findings well describe the clinical characteristics and outcomes in this special population, that will gain more clinical attention, as in- dications for EVT expand. In this context, future studies are needed to validate these findings. Second, the study included patient data from 2010 to 2014. During this period, financial reimbursement for stent retrievers was not permitted and second generation direct aspiration devices were not yet introduced [23]. Thus, outcomes of EVT were somewhat below current standards and this should be taken into account when interpreting the results of this study.

In conclusion, acute ischemic stroke patients with malignant cores between 82 to $209 \mathrm{~mL}$ can be treated with primary neurocritical care based on reperfusion and therapeutic hypothermia, with feasible results. In patients undergoing emergent surgical decompression due to malignant course, the functional outcomes were not satisfactory.

\section{ARTICLE INFORMATION}

\section{Conflict of interest}

No potential conflict of interest relevant to this article.

\section{ORCID}

Seong-Joon Lee, https://orcid.org/0000-0001-9735-6369

Ji Man Hong, https://orcid.org/0000-0001-6803-1207

\section{Author contributions}

Conceptualization: SJL and JMH. Data curation \& Formal analysis: SJL, KSL, JSL, MHC, SEL, and JMH. Visualization \& Writingoriginal draft: SJL and JMH. Writing-review editing: SJL, KSL, JSL, MHC, SEL, and JMH.

\section{REFERENCES}

1. Vahedi K, Hofmeijer J, Juettler E, Vicaut E, George B, Algra A, et al. Early decompressive surgery in malignant infarction of the middle cerebral artery: a pooled analysis of three randomised controlled trials. Lancet Neurol 2007;6:215-22.

2. Hacke W, Kaste M, Bluhmki E, Brozman M, Davalos A, Guidetti D, et al. Thrombolysis with alteplase 3 to 4.5 hours after acute ischemic stroke. N Engl J Med 2008;359:1317-29.

3. Lee JS, Demchuk AM. Choosing a hyperacute stroke imaging protocol for proper patient selection and time efficient endovascular treatment: lessons from recent trials. J Stroke 2015;17:221-8.

4. Hong JM, Lee JS, Song HJ, Jeong HS, Choi HA, Lee K. Therapeutic hypothermia after recanalization in patients with acute ischemic stroke. Stroke 2014;45:134-40.

5. Kollmar R, Schwab S. Therapeutic hypothermia in neurological critical care. Dtsch Med Wochenschr 2010;135:2361-5. 
6. Hwang YH, Jeon JS, Kim YW, Kang DH, Kim YS, Liebeskind DS. Impact of immediate post-reperfusion cooling on outcome in patients with acute stroke and substantial ischemic changes. J Neurointerv Surg 2017;9:21-5.

7. Fuhrer H, Schönenberger S, Niesen WD, Seide S, Meyne J, Gerner ST, et al. Endovascular stroke treatment's impact on malignant type of edema (ESTIMATE). J Neurol 2019;266:223-31.

8. Vahedi K, Vicaut E, Mateo J, Kurtz A, Orabi M, Guichard JP, et al. Sequential-design, multicenter, randomized, controlled trial of early decompressive craniectomy in malignant middle cerebral artery infarction (DECIMAL Trial). Stroke 2007;38:2506-17.

9. Hofmeijer J, Kappelle LJ, Algra A, Amelink GJ, van Gijn J, van der Worp HB, et al. Surgical decompression for space-occupying cerebral infarction (the Hemicraniectomy After Middle Cerebral Artery infarction with Life-threatening Edema Trial [HAMLET] ): a multicentre, open, randomised trial. Lancet Neurol 2009;8:326-33.

10. Thomalla G, Hartmann F, Juettler E, Singer OC, Lehnhardt FG, Köhrmann M, et al. Prediction of malignant middle cerebral artery infarction by magnetic resonance imaging within 6 hours of symptom onset: a prospective multicenter observational study. Ann Neurol 2010;68:435-45.

11. Krieger DW, Demchuk AM, Kasner SE, Jauss M, Hantson L. Early clinical and radiological predictors of fatal brain swelling in ischemic stroke. Stroke 1999;30:287-92.

12. Jeon SB, Koh Y, Choi HA, Lee K. Critical care for patients with massive ischemic stroke. J Stroke 2014;16:146-60.

13. Mayer SA, Coplin WM, Raps EC. Cerebral edema, intracranial pressure, and herniation syndromes. J Stroke Cerebrovasc Dis 1999;8:183-91.

14. Hofmeijer J, Algra A, Kappelle LJ, van der Worp HB. Predictors of life-threatening brain edema in middle cerebral artery infarction. Cerebrovasc Dis 2008;25:176-84.

15. Fiorelli M, Bastianello S, von Kummer R, del Zoppo GJ, Larrue V, Lesaffre E, et al. Hemorrhagic transformation within 36 hours of a cerebral infarct: relationships with early clinical deterioration and 3-month outcome in the European Cooperative Acute Stroke Study I (ECASS I) cohort. Stroke 1999;30:2280-4.

16. Albers GW. Late window paradox. Stroke 2018;49:768-71.

17. Hwang YH, Kang DH, Kim YW, Kim YS, Park SP, Liebeskind DS. Impact of time-to-reperfusion on outcome in patients with poor collaterals. AJNR Am J Neuroradiol 2015;36:495-500.

18. Lee SU, Hong JM, Kim SY, Bang OY, Demchuk AM, Lee JS. Differentiating carotid terminus occlusions into two distinct populations based on Willisian collateral status. J Stroke 2016;18:179-86.

19. Powers WJ, Derdeyn CP, Biller J, Coffey CS, Hoh BL, Jauch EC, et al. 2015 American Heart Association/American Stroke Association focused update of the 2013 guidelines for the early management of patients with acute ischemic stroke regarding endovascular treatment: a guideline for healthcare professionals from the American Heart Association/American Stroke Association. Stroke 2015;46:3020-35.

20. Noorian AR, Rangaraju S, Sun CH, Owada K, Nahab F, Belagaje SR, et al. Endovascular therapy in strokes with ASPECTS 5-7 may result in smaller infarcts and better outcomes as compared to medical treatment alone. Interv Neurol 2015;4:30-7.

21. Boers AMM, Jansen IGH, Beenen LFM, Devlin TG, San Roman $\mathrm{L}$, Heo JH, et al. Association of follow-up infarct volume with functional outcome in acute ischemic stroke: a pooled analysis of seven randomized trials. J Neurointerv Surg 2018;10:1137-42.

22. Hacke W, Schwab S, Horn M, Spranger M, De Georgia M, von Kummer R. 'Malignant' middle cerebral artery territory infarction: clinical course and prognostic signs. Arch Neurol 1996;53:309-15.

23. Lee JS, Lee SJ, Hong JM, Choi JW, Hong JH, Chang HW, et al. Temporal changes in care processes and outcomes for endovascular treatment of acute ischemic stroke: retrospective registry data from three Korean centers. Neurointervention 2018;13:2-12. 